\title{
Minimizing Power Consumption in HetNets with Packet Delay Constraints
}

\author{
Peng-Yong Kong ${ }^{\dagger}$ and George K. Karagiannidis ${ }^{\dagger \ddagger}$ \\ ${ }^{\dagger}$ Department of Electrical and Computer Engineering, \\ Khalifa University of Science, Technology and Research (KUSTAR), Abu Dhabi, United Arab Emirates. \\ ${ }^{\ddagger}$ Department of Electrical and Computer Engineering, Aristotle University of Thessaloniki, \\ Thessaloniki, Greece. \\ Email: \{kongpy, geokarag\}@ieee.org
}

\begin{abstract}
Heterogenous cellular networks can improve network capacity by co-deployment of macro and femto base stations (BSs). The capacity improvement comes with a higher power consumption. This paper proposes a method to minimize the power consumption without compromising the quality-of-service demand which is measured in terms of packet delay. First, we derive a closed-form expression for the packet delay as a function of macro and femto BS densities. Then, we determine the BS densities that minimize the network power consumption under a packet delay constraint. Evaluation results show that heterogeneous networks always outperforms a homogeneous network in terms of power-delay characteristics. With the optimal BS densities for a given packet delay requirement, the minimum power consumption of a heterogeneous network is always lower than that of a homogeneous network.
\end{abstract}

Index Terms-Heterogeneous cellular network; power consumption; packet delay.

\section{INTRODUCTION}

Heterogeneous cellular networks can improve network capacity by deploying overlapping base stations (BSs) in different tiers. In 3GPP [1] and LTE-Advanced [2], femto BSs are deployed within an existing network of macro BSs. The increased capacity is needed to support the rapidly growing network traffic, given the proliferation of broadband mobile applications and smart devices. However, a larger network capacity may require a higher power consumption, and energy cost may constitute up to $50 \%$ of the total operational expenditure of a telecom service provider in some countries [3]. As such, it is important to control power consumption while attaining a higher capacity.

In the literature, Y. Song et al. [4] have proposed a MDP-based vertical handover scheme to minimize power consumption by switching users between macro and femto BSs depending on channel quality and user traffic profile. In separate works, [5] and [6] have proposed different schemes to dynamically activate and deactivate some radio transceivers of a BS to conserve energy depending on the instantaneous traffic load. These transceivers can be the relays or remote radio heads within a heterogeneous network as defined in LTE-Advanced. Furthermore, J. Kim et al. have proposed in [7] a scheme to dynamically turn off some BSs when the respective cells have low traffic load. This is to save energy, and the coverage of the turned-off cell is provided by uneven expansion of neighbor cells. Compared to the aforementioned works, this paper aims to minimize the power consumption of a heterogeneous cellular network without compromising its quality-of-service (QoS), which is given in terms of packet delay. Specifically, we aim to reduce transmission power to conserve energy although it is only a fraction of the overall power consumption of a communication network.

By employing stochastic geometry, [8] has developed a model for the downlink of a heterogeneous network. Based on this model, [8] has further provided a closed-form expression for the outage probability as a function of BS densities, transmission powers and signal-to-interference ratio (SIR) thresholds at different tiers. Building on the works in [8], [9] has introduced association weight as a control parameter in achieving optimal rate coverage. By adjusting the weights, the amount of traffic being offloading to a particular tier of a heterogeneous network can be influenced. Following [9], the authors in [10] have also considered shadowing effects as the signal propagation impairments in addition to path loss and Rayleigh fading. This collection of works offer a good foundation in understanding the performance of a heterogeneous network. However, they have not dealt with the important issue of power consumption and energy efficiency. In a separate effort, [11] has determined the optimal heterogeneous BS densities that will minimize the power consumption subject to a QoS requirement in terms of data rate over a downlink. But the data rate does not take into account queueing delay and a user may not transmit over the link all the times. In this case, packet delay appears as a better QoS measure. With M/G/1 queuing analysis, [12] has developed a power-delay relationship for downlink transmission in heterogeneous networks. Importantly, [12] shows that there is trade-off between power consumption and packet delay. However, similar to [11], there is no simple closed-form expression for the power-delay relationship in [12] making it difficult for use to minimize power consumption.

In this paper, we derive a closed-form expression for the packet delay as a function of heterogeneous BS densities. Then, we propose a method to determine the BS densities in order to minimize power consumption given a packet delay 
requirement. The rest of this paper is organized as follows. In Section II, we present the system model. Section III provides the detailed method in finding the optimal BS densities in minimizing power consumption. We present and discuss some numerical results in Section IV, before ending the paper with concluding remarks in Section V.

\section{SYSTEM MODEL}

We consider a two-tier heterogeneous cellular network, where tier-1 is for macro BSs, and tier-2 is for femto BSs. Let $P_{i}$ be the transmission power of an $i$-th tier BS, with $i=\{1,2\}$. Reasonably, $P_{1}>P_{2}$ such that the coverage area for a macro BS is larger than that of a femto BS. The transmission power $P_{i}$ is fixed and no dynamic power control is implemented. Similar to [8], [11] and [12], we assume that the BSs in each tier are located in an Euclidean plane according to an independent Poisson Point Process (PPP), with $\rho_{i}$ being the BS density of the $i$-th tier. We also assume that users are randomly located in the same plane, according to a PPP with density $\rho_{u}$.

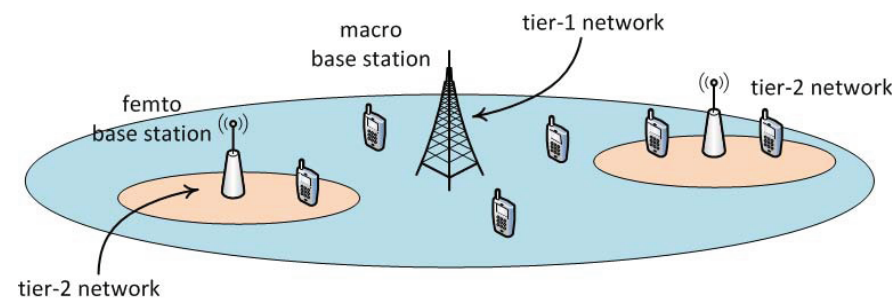

Fig. 1. System model of a two-tier heterogeneous cellular network. Macro and femto base stations form tier-1 and tier-2, respectively.

\section{Minimize Power Consumption Under a Packet DELAY CONSTRAINT}

Let $\gamma\left(x_{i}\right)$ be the downlink SIR at a randomly located user for the signal received from an $i$-th tier BS which is positioned at point $x_{i} \in \mathcal{X}_{i}$, where $\mathcal{X}_{i}$ is the set of all locations of $i$-th tier BSs. The user is connected to the $i$-th tier BS if $\gamma\left(x_{i}\right)>$ $\beta_{i}$, where $\beta_{i}$ is the SIR threshold for acceptable QoS. Let each user be connected to only one BS that has the maximum SIR among all tiers, all the channels suffer from independent Rayleigh fading, and SIR threshold $\beta_{i}>1$ for each $i$-th tier. Then, according to [8], the probability of a user be connected to any BS from any tier can be written as

$$
\mathbb{P}\left\{\bigcup_{\substack{x_{i} \in \mathcal{X}_{i}, i=\{1,2\}}} \gamma\left(x_{i}\right)>\beta_{i}\right\}=\frac{\pi}{C(\alpha)} \frac{\sum_{i=1}^{2} \rho_{i} P_{i}^{2 / \alpha} \beta_{i}^{-2 / \alpha}}{\sum_{i=1}^{2} \rho_{i} P_{i}^{2 / \alpha}},
$$

where $\alpha>2$ is the path loss exponent, $C(\alpha)$ is a constant determined as $C(\alpha)=\frac{2 \pi^{2}}{\alpha} \csc \left(\frac{2 \pi}{\alpha}\right)$, and $\mathbb{P}\{\cdot\}$ denotes probability. Let $\beta$ be the same SIR threshold adopted for all tiers. Then, the outage probability can be determined as the compliment of (1). Note that we assume successful packet transmission if there is no outage event occurs during the transmission. Then, the probability of a successful packet transmission is given as

$$
P_{s}=\frac{\pi}{C(\alpha) \beta^{2 / \alpha}} .
$$

When a packet is not transmitted successfully, it is retransmitted. Let $n$ be the number of re-transmission attempts before a packet is delivered successfully. Then, when a user is attached to an $i$-th tier BS, the average packet delay is

$$
\overline{\mathcal{D}}_{i}=E\left[\frac{(n+1) L}{R_{i}}\right],
$$

where $L$ is the packet size and $R_{i}$ is the transmission rate from an $i$-th tier BS. In order to achieve the desired transmission success probability, the value of $R_{i}$ should be determined based on $\beta$ as:

$$
R_{i}=\frac{W_{i}}{N_{i}+1} \log _{2}(1+\beta),
$$

where $W_{i}$ is the bandwidth available at an $i$-th tier BS, and $N_{i}$ is the number of users connected to an $i$-th tier BS. Here, $W_{i} /\left(N_{i}+1\right)$ is the bandwidth per user, where the term " +1 " takes into account the share of bandwidth for the BS. Notice that the number of users attached to an $i$-th tier BS can be different from one BS to another. We assume random variable $N_{i}$ is independent from $n$. Applying (4) into (3),

$$
\begin{aligned}
\overline{\mathcal{D}}_{i} & =\frac{E\left[(n+1)\left(N_{i}+1\right)\right] L}{W_{i} \log _{2}(1+\beta)} \\
& =\left(\sum_{n=0}^{\infty}\left(1-P_{s}\right)^{n} n+\sum_{n=0}^{\infty}\left(1-P_{s}\right)^{n}\right) \frac{E\left[N_{i}+1\right] P_{s} L}{W_{i} \log _{2}(1+\beta)} \\
& =\frac{E\left[N_{i}+1\right] L}{P_{s} W_{i} \log _{2}(1+\beta)} .
\end{aligned}
$$

Since users are distributed within a network following PPP, finding the average number of users attached to an $i$-th tier BS requires us to first find the area of the cell. With PPP, the cell geometry in a heterogeneous network follows a multiplicatively weighted Poisson Voronoi (MWPV) tessellation [14]. MWPV tessellation is different from classical Poisson Voronoi tessellation in the sense that distance of a cell boundary from a BS is multiplied by a positive weight which is determined by the BS type. In the literature, [11] has adopted a simulation plus curve-fitting method to determine the cell size distribution for a two-tier heterogeneous network. This method is reasonable but a new set of simulations and curve-fittings is needed whenever there is a change in system parameters, resulting in no possible closed-form expression for packet delay.

We have two observations on MWPV tessellation. First, Ferenc et al. [13] have discovered an accurate and universal distribution function for normalized cell sizes of a classical Poisson Voronoi tessellation. The accuracy of the distribution function has been verified against the findings in [14]. The distribution function is dependent only on the Poisson point density. Second, in forming the Poisson Voronoi tessellation for a heterogeneous network, the cell radius of an $i$-th tier 
BS will grow at a speed proportional to $P_{i}^{1 / \alpha}$. Base on these two observations, we will be able to adopt the existing cell area distribution function from [13] as long as we can find an equivalent homogeneous BS density for a heterogeneous network.

We assume the area of a cell is proportional to the square of its radius. Since $P_{1}>P_{2}$, the area of a macro cell will grow $\left(P_{1} / P_{2}\right)^{2 / \alpha}$ times faster than that of a femto cell in forming a Poisson Voronoi tessellation. As such, we need to combine as many as $\left(P_{1} / P_{2}\right)^{2 / \alpha}$ femto cells to achieve the same cell area grow rate of a macro cell. Therefore, in the presence of both macro and femto BSs, a heterogeneous network is equivalent to a homogeneous network deployed with only macro BSs with an equivalent macro BS density $\hat{\rho}_{1}$ determined as

$$
\hat{\rho}_{1}=\rho_{1}+\left(\frac{P_{1}}{P_{2}}\right)^{-\frac{2}{\alpha}} \rho_{2} .
$$

Similarly, a heterogeneous network is equivalent to a homogeneous network deployed with only femto BSs with an equivalent femto BS density $\hat{\rho}_{2}$ determined as

$$
\hat{\rho}_{2}=\left(\frac{P_{1}}{P_{2}}\right)^{\frac{2}{\alpha}} \rho_{1}+\rho_{2}
$$

In (7), the multiplicative factor $\left(P_{1} / P_{2}\right)^{2 / \alpha}$ takes into account the fact that the size of each macro cell grows faster than a single femto cell. Although developed separately, (7) is the same as a similar expression in [11] and this serves as a good verification.

Let $\mathcal{A}_{i}$ be a random variable representing the cell size of an $i$-th tier BS. Then, following the two observations presented above, the probability density function for $\mathcal{A}_{i}$ is given as

$$
f_{\mathcal{A}_{i}}(a)=d \hat{\rho}_{i}^{7 / 2} a^{5 / 2} e^{-(7 / 2) \hat{\rho}_{i} a},
$$

where constant $d=\frac{343}{15} \sqrt{\frac{7}{2 \pi}}$ has been determined and verified by [13]. In the literature, [9] has derived based on [13] a different distribution function for cell sizes. In [9], cell size distribution depends on the ratio of BS density over probability of user associated with such a BS. Finding the ratio involves further derivations leading to an expression (i.e., equation (11) in [9]) that has different coefficients compared to (8). The expression in [9] is more complicated because it is more general, considering more than 2 tiers and capturing various controllable parameters such as association weights. Also, [9] considers a case where a selected BS has a larger area than a typical one. Although (8) is limited to a two-tier network, we continue to use it for its simplicity and intuitive derivation based on equivalent density we introduced in (6) and (7).

Fig. 2(a) shows a sample of the cell size distributions obtained using (8). It is reasonable that a femto cell has a smaller size compared to a macro cell. Fig. 2 is presented for the purpose of verification against similar distributions in [11] and [12] that are obtained without the closed-form expression of cell size distribution. With the cell size distribution, we can now determined the probability mass function for number of

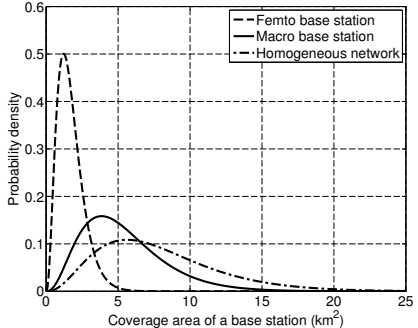

(a) $f_{\mathcal{A}_{i}}(a)$

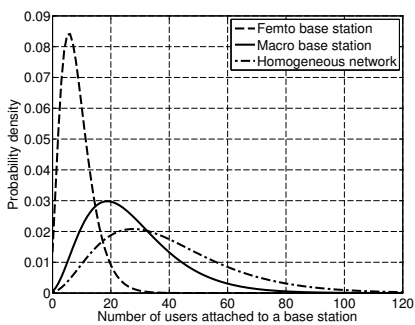

(b) $P_{N_{i}}(n)$
Fig. 2. (a) is the probability density functions for cell size and (b) is the probability mass function for number of attached users. Both are for $\rho_{u}=5.0$, $\rho_{1}=0.1, \rho_{2}=0.27, P_{1}=10 \mathrm{Watt}$ and $P_{2}=1$ Watt. For the homogeneous network, $\rho_{2}$ and $P_{2}$ are zero.

users attached to an $i$-th tier BS as

$$
\begin{aligned}
P_{N_{i}}(n) & =\int_{0}^{\infty} P\left\{N_{i}=n \mid a\right\} f_{\mathcal{A}_{i}}(a) d a \\
& =\int_{0}^{\infty} \frac{\left(\rho_{u} a\right)^{n}}{n !} e^{-\rho_{u} a} f_{\mathcal{A}_{i}}(a) d a \\
& =\frac{\rho_{u}^{n} d \hat{\rho}_{i}^{7 / 2}}{n !} \Gamma\left(\frac{7}{2}+n\right)\left(\rho_{u}+\frac{7}{2} \hat{\rho}_{i}\right)^{-(7 / 2+n)}
\end{aligned}
$$

Fig. 2(b) shows a sample of the probability mass function for number of users in a cell. Compared to heterogeneous cells, a homogeneous network has a wider spread of user numbers consistent with its larger cell size. Despite a slight difference in values, Fig. 2(b) has a same shape as the similar figure in [12]. The difference in values is due the adoption of (8) in this paper. This offers a good verification to our derivation.

For each of the $i$-th tier, we define a variable $r_{i}=\rho_{u}\left(\rho_{u}+\right.$ $\left.\frac{7}{2} \hat{\rho}_{i}\right)^{-1}$. Here, $r_{i}$ does not have a physical meaning and it is introduced to simplify expression. Using $r_{i}$ and (9), we can determine the average number of users in an $i$-th tier cell as

$$
\begin{aligned}
E\left[N_{i}\right] & =\sum_{n=0}^{\infty} n P_{N_{i}}(n) \\
& =d\left[\frac{2}{7}\left(1-r_{i}\right)\right]^{7 / 2} \sum_{n=0}^{\infty} \frac{n}{n !} \Gamma\left(\frac{7}{2}+n\right) r_{i}^{n} \\
& =d\left[\frac{2}{7}\left(1-r_{i}\right)\right]^{7 / 2} \frac{105 \sqrt{\pi} r_{i}}{16\left(1-r_{i}\right)^{9 / 2}}=\frac{\rho_{u}}{\hat{\rho}_{i}} .
\end{aligned}
$$

Equation (10) indicates that the average number of users in a cell is simply the ratio of user density to equivalent BS density. Increasing user density increases the number of user in each cell. On the other hand, increasing BS density results in fewer users being served by each BS.

Applying (10) to (5) gives the average packet delay in an $i$-th tier cell as

$$
\overline{\mathcal{D}}_{i}=\frac{\left(\rho_{u}+\hat{\rho}_{i}\right) L}{P_{s} \hat{\rho}_{i} W_{i} \log _{2}(1+\beta)} .
$$

A randomly selected user can be in either a tier- 1 or tier-2 cell. The probability of a random user being in an $i$-th tier cell depends on the fraction of total network area covered by all 
$i$-th BSs. Therefore, the network wide average packet delay $\overline{\mathcal{D}}$ is given as

$$
\overline{\mathcal{D}}=\frac{\rho_{1} E\left[\mathcal{A}_{1}\right] \overline{\mathcal{D}}_{1}}{\rho_{1} E\left[\mathcal{A}_{1}\right]+\rho_{2} E\left[\mathcal{A}_{2}\right]}+\frac{\rho_{2} E\left[\mathcal{A}_{2}\right] \overline{\mathcal{D}}_{2}}{\rho_{1} E\left[\mathcal{A}_{1}\right]+\rho_{2} E\left[\mathcal{A}_{2}\right]},
$$

where the average area of an $i$-th tier cell is determined using (8) as

$$
\begin{aligned}
E\left[\mathcal{A}_{i}\right] & =\int_{0}^{\infty} a f_{\mathcal{A}_{i}}(a) d a=d \hat{\rho}_{i} \int_{0}^{\infty} a^{7 / 2} e^{-(7 / 2) \hat{\rho}_{i} a} d a \\
& =\Gamma\left(\frac{9}{2}\right) \frac{d}{\hat{\rho}_{i}}\left(\frac{7}{2}\right)^{-9 / 2}=\frac{1}{\hat{\rho}_{i}} .
\end{aligned}
$$

Combining (11), (12) and (13), we have the following closed-form expression for the average packet delay in a heterogeneous network

$$
\begin{aligned}
\overline{\mathcal{D}}= & \frac{L \beta^{2 / \alpha} C(\alpha)}{\pi\left(\rho_{1} \hat{\rho}_{2}+\rho_{2} \hat{\rho}_{1}\right) \log _{2}(1+\beta)}\left(\frac{\rho_{1} \hat{\rho}_{2}\left(\rho_{u}+\hat{\rho}_{1}\right)}{W_{1} \hat{\rho}_{1}}\right. \\
& \left.+\frac{\rho_{2} \hat{\rho}_{1}\left(\rho_{u}+\hat{\rho}_{2}\right)}{W_{2} \hat{\rho}_{2}}\right) .
\end{aligned}
$$

Let network power consumption in terms of Watt per $\mathrm{Km}^{2}$ be determined as $\mathcal{P}=\rho_{1} P_{1}+\rho_{2} P_{2}$. Then, we want to find a set of BS densities that minimizes the power as

$$
\begin{array}{ll} 
& \min _{\rho_{1}, \rho_{2}}\left\{\mathcal{P}=\rho_{1} P_{1}+\rho_{2} P_{2}\right\} \\
\text { s.t. } & \overline{\mathcal{D}} \leq \mathcal{D}^{*} \\
& \rho_{1} \mathcal{C}_{1}+\rho_{2} \mathcal{C}_{2} \leq \mathcal{C}^{*} \\
& \rho_{1} \geq \rho_{1}^{*},
\end{array}
$$

where $\mathcal{D}^{*}$ is the packet delay requirement, and $\rho_{1}^{*}$ is the lower limit in macro cell density. Also, $\mathcal{C}_{1}$ and $\mathcal{C}_{2}$ are the costs for each tier- 1 and tier-2 BS, respectively. The cost constraint $\mathcal{C}^{*}$ is determined as

$$
\mathcal{C}^{*}=\left(\frac{\rho_{u} L \beta^{2 / \alpha} C(\alpha)}{\mathcal{D}^{*} \pi W_{1} \log _{2}(1+\beta)-L \beta^{2 / \alpha} C(\alpha)}\right) \mathcal{C}_{1} .
$$

Here, $\mathcal{C}^{*}$ is given by the cost to operate a homogeneous macro cell network in achieving the same packet delay requirement. As such, we requires the heterogeneous network to be more cost effective than a macro-only network while achieving a minimum power consumption. We have solved the formulated optimization problem using Matlab's optimization toolbox which is capable of dealing with non-linear constraints.

\section{Numerical Results}

We present and discuss numerical results in this section. These results are collected with the following parameter settings unless specified otherwise: $\alpha=4, \beta=1.0, \rho_{u}=100$, $P_{1}=10$ Watt, $P_{2}=1$ Watt, $L=800$ symbols, $W_{1}=10$ $\mathrm{MHz}, W_{2}=20 \mathrm{MHz}, \rho_{1}^{*}=0.04, \mathcal{C}_{1}=10$ and $\mathcal{C}_{2}=5$.

Fig. 3 shows the power-delay characteristics for heterogeneous networks. In these figures, $\rho_{1}=0.1$ and $\rho_{2}$ is varied to achieve different power consumption levels. We present these results to show their consistency with the results in [12]. This is a verification of reasonability of the derived closed-form expression. Similar to [12], our results show that heterogeneous networks exhibit far better power-delay characteristics compared to a homogeneous network with only macro BSs. Specifically, heterogeneous network can achieve a lower power consumption given a packet delay or a smaller packet delay given a power consumption.

Comparing Fig. 3(a) and (b), we see that a higher user density leads to a higher packet delay. This is because of a larger number of users attached to a BS resulting in a smaller share of bandwidth for each user. Comparing Fig. 3(a) and (c), we notice that a higher SIR threshold $(\beta)$ leads to a lower packet delay. Although a higher $\beta$ can result in a higher outage probability, this negative effect is less significant than an increased spectral efficiency attained by a larger $\beta$. This implies $\beta$ may be an optimization parameter that we can exploit to minimum network power consumption.

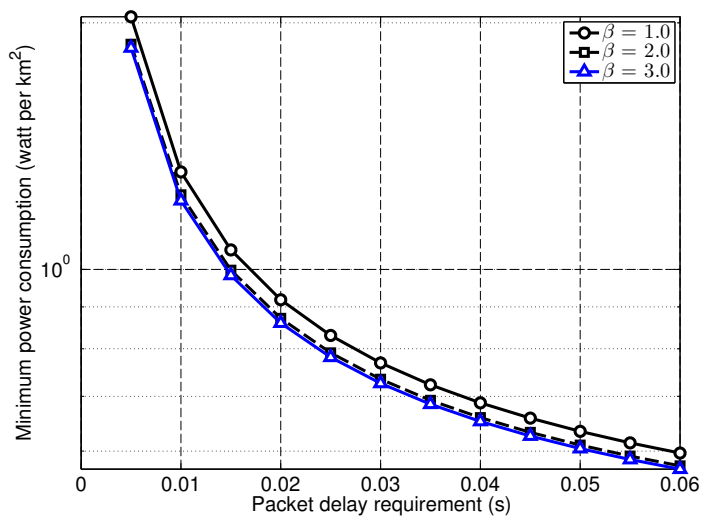

Fig. 4. Minimum power consumption given a packet delay requirement.

Fig. 4 shows the minimum power consumption for a heterogeneous network given a packet delay requirement. Comparing Fig. 3 and Fig. 4, the attained minimum power consumption is always lower than that of an arbitrary setting of a same heterogeneous network. For example, for average packet delay of $10 \mathrm{~ms}$, the minimum power consumption is 1.3 Watt per $\mathrm{Km}^{2}$ while it is about 2.2 Watt per $\mathrm{Km}^{2}$ in Fig. 3(b). By achieving the minimum power consumption, heterogeneous network can achieve a power saving of more than 50\% compared to a homogeneous network with only macro BSs. For example, given a packet delay requirement of $60 \mathrm{~ms}$ with $\beta=1.0$, the power consumptions are 0.6 and 2.1 Watt per $\mathrm{Km}^{2}$ for heterogeneous network and homogeneous macro network, respectively. The percentage of power saving is lower with a larger packet delay requirement. This is because the homogeneous BS density in such a less stringent condition is already low leading to a limited room for improvement. At a large delay requirement, power consumption of a heterogeneous network approaches that of a homogeneous network with only femto cells. This is because at such a setting, the macro BS density is very low compared to the femto BS density, after greedily deploying femto BSs that consume only a tenth of the power. 


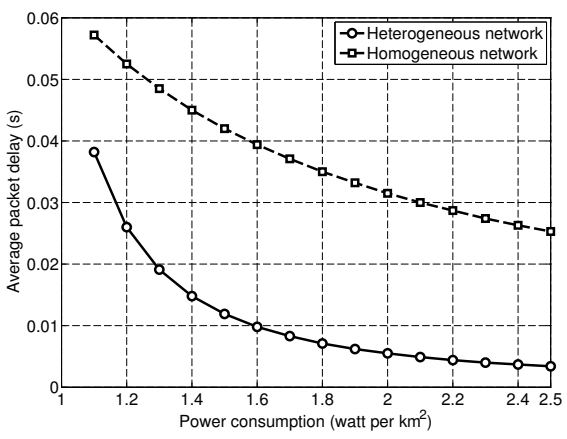

(a) $\beta=1.0$, and $\rho_{u}=50$.



(b) $\beta=1.0$, and $\rho_{u}=100$.

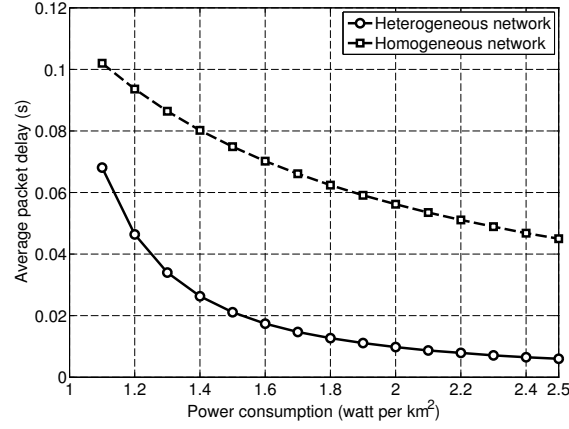

(c) $\beta=2.0$, and $\rho_{u}=100$.

Fig. 3. Power consumption and packet delay characteristics for heterogeneous and homogeneous networks. For heterogeneous networks, $\rho_{1}=0.1$ and $\rho_{2}$ is the variable used to control the power consumption. For homogeneous networks, $\rho_{2}$ and $P_{2}$ are both zero.

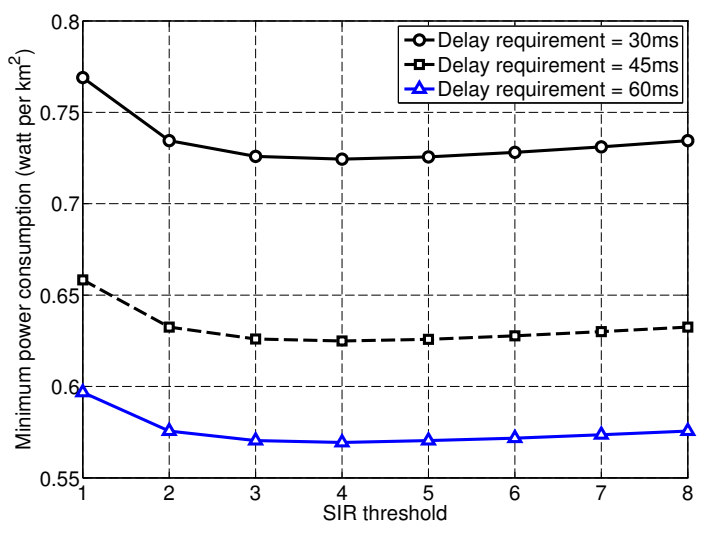

Fig. 5. Minimum power consumption at different SIR thresholds, $\beta$.

Fig. 4 also reveals that adding femto BSs can help in meeting a packet delay requirement which is otherwise infeasible with only macro BSs. For example, it is impossible to achieve 20 ms packet delay for the homogeneous network in Fig. 3(b). However, Fig. 4 shows that 20 ms packet delay can be reached comfortably with $\beta=1.0$. While increasing $\beta$ from 1.0 to 2.0 gives a noticeable reduction in power consumption, further increase in $\beta$ does not yield any significant improvement. As explained earlier, this is because a higher $\beta$ causes a higher outage probability. Fig. 5 shows that there exits an optimal $\beta$ in achieving a minimum power consumption. After careful examination, as indicated in the figure, the optimal $\beta$ is 3.92 and is not affected by $\mathcal{D}^{*}$. As such, there is no need to include $\beta$ as an additional control parameter in the optimization problem formulated above.

\section{CONCLUSION}

We have developed a closed-form expressions for packet delay in a heterogeneous network. We have formulated an optimization problem to determine the macro and femto BS densities that minimize power consumption given a packet delay requirement and a cost constraint. We have solved the optimization problem using Matlab's optimization tool- box. Numerical results confirm that the derived expression is consistent with existing findings in the literature. Given a delay requirement, the optimal BS densities can reduce heterogeneous network power consumption by more than $50 \%$ compared to a homogeneous network with only macro BSs.

\section{REFERENCES}

[1] A. Damnjanovic, J. Montojo, Y. Wei, T. Ji, T. Luo, M. Vajapeyam, T. Yoo, O. Song and D. Malladi, "A survey on 3GPP heterogeneous networks," IEEE Comms. Mag., vol. 18, no. 3, pp. 10 - 21, June 2011.

[2] A. Khandekar, N. Bhushan, J. Tingfang and V. Vanghi, "LTE-Advanced: Heterogeneous networks," in Proc. European Wireless Conference, April 2010, pp. $978-982$.

[3] S. Tombaz, M. Usman and J. Zander, "Energy effciency improvements through heterogeneous networks in diverse traffc distribution scenarios," in Proc. Int. Conf. Communications and Networking in China, August 2011, pp. $708-713$.

[4] Y. Song, P.-Y. Kong and Y. Han, "Power-optimized vertical handover scheme for heterogeneous wireless networks," IEEE Communications Letters, vol. 18, no. 2, pp. 277 - 280, February 2014.

[5] P.-Y. Kong, "Optimal probabilistic policy for dynamic resource activation using Markov decision process in green wireless networks," IEEE Trans. Mobile Computing, 2014.

[6] P.-Y. Kong and D. Panaitopol, "Reinforcement learning approach to dynamic activation of base station resources in wireless networks," in Proc. IEEE Int. Symposium on Personal, Indoor, and Mobile Radio Communications (PIMRC), September 2013, pp. 3264 - 3268.

[7] J. Kim, P.-Y. Kong, N.-O. Song, J.-K. K. Rhee and S. A.-Araji, "MDPbased dynamic base station management for power conservation in self-organizing networks," in Proc. IEEE Wireless Communications and Networking Conference (WCNC), April 2014.

[8] H. S. Dhillon, R. K. Ganti, F. Baccelli and J. G. Andrews, "Modeling and analysis of K-tier downlink heterogeneous cellular networks," IEEE Jnl. Sel. Areas Communications, vol. 30, no. 3, pp. 550 - 560, April 2012.

[9] S. Singh, H. S. Dhillon and J. G. Andrews, "Offloading in heterogeneous networks: Modeling, analysis and design insights," IEEE Trans. Wireless Communications, vol. 12, no. 5, pp. 2484 - 2497, May 2013.

[10] H. S. Dhillon and J. G. Andrews, "Downlink rate distribution in heterogeneous cellular networks under generalized cell selection," 2013, arXiv: 1306.6122

[11] D. Cao, S. Zhou and Z. Niu, "Optimal base station density for energyefficient heterogeneous cellular networks", in Proc. IEEE ICC, June 2012 , pp. $5890-5894$.

[12] P.-Y. Kong, "Power consumption and packet delay relationship for heterogeneous wireless networks," IEEE Communications Letters, vol. 17, no. 7 , pp. 1376 - 1379, July 2013.

[13] J.-S. Ferenc and Z. Neda, "On the size-distribution of Poisson Voronoi cells", February 2008, arXiv:cond-mat/0406116v2.

[14] M. Tanemura, "Statistical distributions of Poisson Voronoi cells in two and three dimensions", Forma, vol. 18, pp. 221 - 247, November 2003. 